\title{
Group Contribution Method to Predict the Mass Transfer Coefficients of Organics through Various RO Membranes
}

Supporting Information

Contents:

6 Texts

8 Tables

6 Figures

Ryan Kibler ${ }^{1}$, Benjamin Mohrhardt ${ }^{1}$, Muxue Zhang ${ }^{1}$, Lauren Breitner ${ }^{2}$, Kerry J. Howe ${ }^{3}$, and Daisuke Minakata ${ }^{1 *}$

1. Department of Civil and Environmental Engineering, Michigan Technological University, 1400 Townsend Drive, Houghton, Michigan, 49931

2. Trussell Technologies, Inc. San Diego, California, 92075

3. Department of Civil, Construction and Environmental Engineering, University of New Mexico, MSC01 1070, Albuquerque, New Mexico, 87131-1070

* corresponding author: dminakat@mtu.edu; 1400 Townsend Drive, Houghton, Michigan 49931 U.S.A.; 906-487-1830. 


\section{Text S1: Experimental procedures and properties of membrane products}

The thorough experimental description and membrane properties are found in our previous publication ${ }^{1}$. Our membrane products included four brackish water (BW) membranes such as ESPA2-LD by Hydranautics, TMG(D) by Toray, BW30XFRLE by Dow Filmtec, and AG-LF by GE, and two seawater (SW) membranes such as SW30XHR by Dow Filmtec and TM800M by Toray. Typical relationship between rejection of sodium chloride and calculated mass transfer coefficients values for ultralow pressure, BW and SW membranes are shown in Figure S1. Briefly, the BW membranes were tested at 5.2, 10.3, and 15.5 bar, and the SW membranes were tested at $10.3,15.5$, and 24.1 bar to provide a range of operating conditions using a bench-top experimental apparatus using flat sheets BW and SW membranes. The water flux, $\mathrm{k}_{\mathrm{w}}$, ranged from 11 to $74 \mathrm{~L} / \mathrm{m}^{2}-\mathrm{h}$ for the BW membranes and 6 to $50 \mathrm{~L} / \mathrm{m}^{2}$-h for the $\mathrm{SW}$ membranes. The rejection of a suite of low molecular weight organic compounds was determined. All of the organic compounds were added to the tank with buffered DI water. The spike concentration of each organic compound depended on the expected rejection. At the end of the 72-hour equilibration period, feed and permeate samples were taken at all three test pressures for each group of membranes, with a minimum of an hour of operation at each pressure. A nominal cross flow velocity of $0.25 \mathrm{~m} / \mathrm{s}$ was maintained throughout the experiments, which corresponds to typical operating conditions in full-scale RO elements. The feed solutions were kept at a constant $\mathrm{pH}$ of 6.5 by adding $1.6 \mathrm{mM}$ sodium phosphate and $0.4 \mathrm{mM}$ disodium phosphate. The $\mathrm{pH}$ of 6.5 was chosen based on the $\mathrm{pH}$ generally used in reuse practices, as well as to ensure all organics tested were neutral. Trace organic concentrations were determined by a commercial laboratory using EPA Method 5030 (Purge and Trap) for extraction ${ }^{2}$ and EPA Method 8260 (Volatile Organic Compound Analysis by GC/MS) for quantification ${ }^{3}$. 


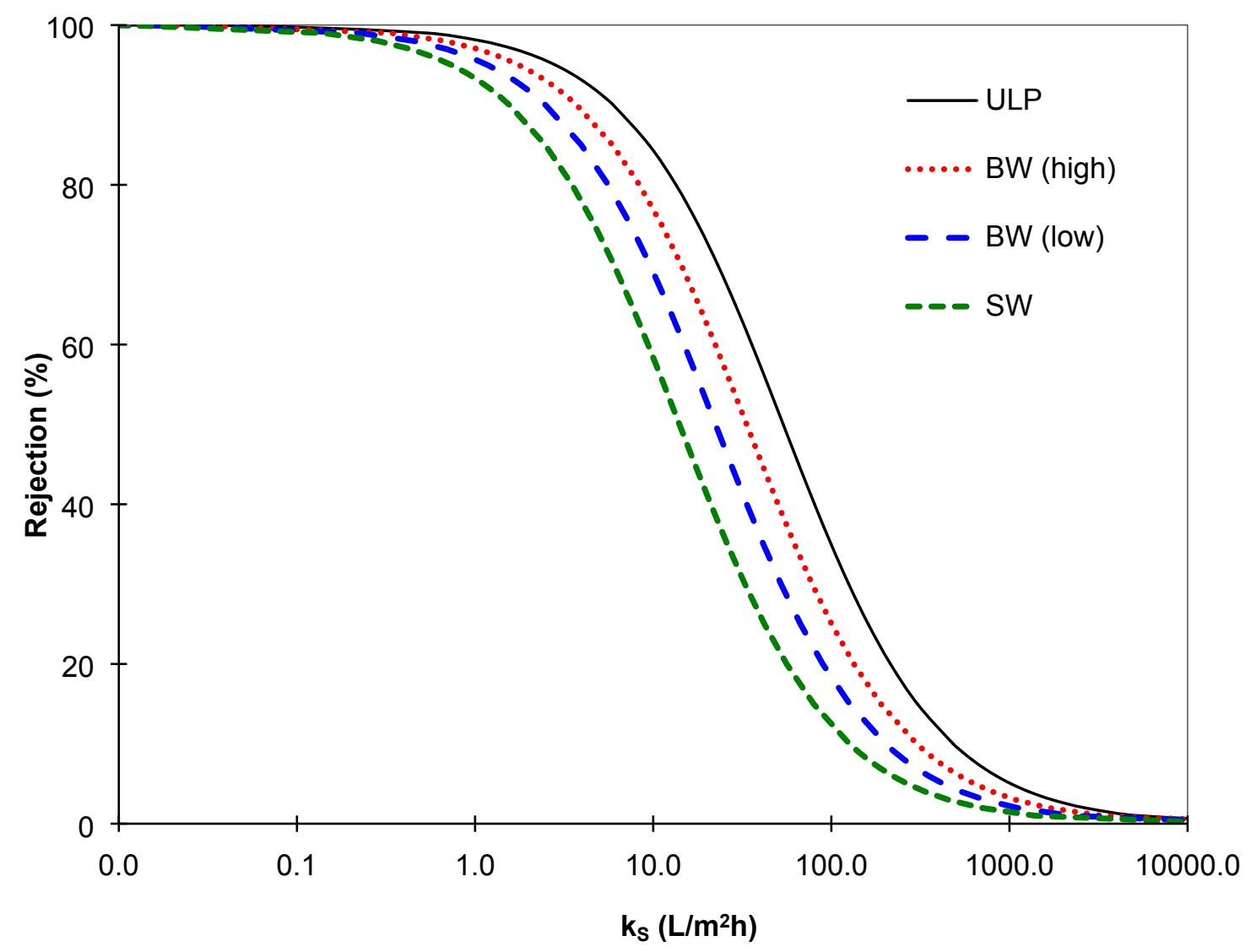

Figure S1: Relationships between rejection of sodium chloride and calculated $\mathrm{k}_{\mathrm{s}}$ values for ultralow pressure (UPR), seawater (SW), and brackishwater (BW) membranes.

\section{Text S2: Determination of mass transfer coefficients from experiments}

The rejection of organics by RO can be quantified using the ratio of organic concentrations in permeate, $C_{\mathrm{p}}$, and feed solution, $C_{\mathrm{F}}$, as shown in equations (S1 and $\left.\mathrm{S} 2\right)^{4}$

Rej $=1-\frac{C_{\mathrm{P}}}{C_{\mathrm{F}}}$

where $C_{\mathrm{p}}$ is the ratio of the flux of organic compound, $J_{\text {Organic }}$, to that of water, $J_{\mathrm{w}}$, and can be expressed as 
$C_{P}=\frac{J_{\text {Organic }}}{J_{\mathrm{W}}}=\frac{k_{\text {Organic }}\left(\beta C_{\mathrm{F}}-C_{\mathrm{P}}\right)}{k_{\mathrm{W}}\left[\Delta P-\left(\beta \Pi_{\mathrm{F}}-\Pi_{\mathrm{P}}\right)\right]}$

where $k_{\text {Organic }}$ and $k_{\mathrm{W}}$ are the organic compound and water mass transfer coefficients, respectively, $\beta$ is the concentration polarization factor, $\Delta \mathrm{P}$ is the pressure applied across the membrane, $\Pi_{F}$ and $\Pi_{P}$ are the osmotic pressures of feed and permeate solutions. Given that $\beta$, $\Delta \mathrm{P}, \Pi_{\mathrm{F}}$ and $\Pi_{\mathrm{P}}$ are determined by the operating conditions and $k_{\mathrm{W}}$ is a membrane property, $k_{\text {Organic }}$ is the fundamental property that reflects the rejection of a target organic compound. The $\beta$ was calculated by equation (S3) and the concentration polarization mass transfer coefficient, $\mathrm{m} / \mathrm{s}$, is calculated using a correlation shown in equation (S4) regardless the external experimental studies were conducted at pilot or bench scale to avoid the overestimation of a concentration polarization factor, $\beta .^{8}$

$$
\begin{aligned}
& \beta=(1-\operatorname{Rej})+\operatorname{Rej}\left(e^{J_{\mathrm{w}} / k_{\mathrm{CP}}}\right) \\
& k_{\mathrm{CP}}=\lambda \frac{D_{L}}{d_{H}}(\mathrm{Re})^{0.5}(\mathrm{Sc})^{1 / 3}
\end{aligned}
$$

where $\lambda$ ranges from 0.40 to 0.54 for different elements, $D_{\mathrm{L}}$ is the diffusion coefficient for solute in water, $\mathrm{m}^{2} / \mathrm{s}, d_{\mathrm{H}}$ is the hydraulic diamter $\left(=\frac{4(\text { volume of flow channel })}{\text { wettedsurface }}\right), \operatorname{Re}$ is the Reynolds number $\left(=\frac{\rho v d_{\mathrm{H}}}{\mu}\right), \mathrm{Sc}$ is the Schmidt number $\left(=\frac{\mu}{\rho D_{\mathrm{L}}}\right), \rho$ is the feed water density, $\mathrm{kg} / \mathrm{m}^{3}, v$ is the velocity in feed channel, $\mathrm{m} / \mathrm{s}, \mu$ is the feed water dynamic viscosity, $\mathrm{kg} / \mathrm{m} \mathrm{s}$. The calculated $\beta$ values for each membrane product is summarized in Table S1 below. 


\section{Text S3: Screening procedure of $\mathrm{k}_{\text {Organic }}$ values}

The rejection is approximately $98 \%$ when the $k_{\text {Organic }}$ value was up to $1 \mathrm{~L} / \mathrm{m}^{2}-\mathrm{h}$. Organic compounds with greater than $98 \%$ (i.e., below $1 \mathrm{~L} / \mathrm{m}^{2}-\mathrm{h}$ ) rejection are not important to include in the model development because it can be concluded that the rejection of organic compounds is excellent. On the other end, accurately predicting the rejection of organic compounds is not important when the rejection is below $20 \%$, because it can be concluded that the rejection is poor and utilities would know that they cannot rely on RO for any appreciable removal of organic compounds.

Although the $k_{\text {Organic }}$ values smaller than $1 \mathrm{~L} / \mathrm{m}^{2}$-h were not included for the GCM development through $\mathrm{BW}$ membranes due to the reasons described above, these data were included through the SW membranes because the performance of SW is superior to that of BW and many organic rejections showed greater than $97 \%$. For the BW membranes, $20 \%$ of the rejection corresponds to the $k_{\text {Organic }}$ values in the range from 90 to $135 \mathrm{~L} / \mathrm{m}^{2}-\mathrm{h}$. Thus, it is concluded to exclude any data greater than $100 \mathrm{~L} / \mathrm{m}^{2}-\mathrm{h}$ of $k_{\text {Organic }}$ values from the development of a GCM.

\section{Text S4: Procedure to calculate the rejections from the $\mathrm{k}_{\text {Organic }}$ values}

To calculate the rejection based on the $\mathrm{k}_{\text {Organic }}$ values, the concentration polarization factor $(\beta)$, volumetric flux of water $\left(\mathrm{J}_{\mathrm{w}}\right)$ for each membrane, the following equation and values from Table S1 were used:

$$
\operatorname{Rej}_{c a l}(\%)=\frac{1}{\frac{k_{s} \beta}{J_{w}}+1}
$$


Table S1: Experimentally obtained medium pressure data for each RO membrane

\begin{tabular}{|c|c|c|}
\hline Membrane & $\begin{array}{c}\text { Concentration Polarization } \\
\text { Factor, } \beta_{0}\end{array}$ & $\begin{array}{c}\text { Volumetric Water Flux, } \\
\mathrm{J}_{\mathrm{w}}\left(\mathrm{L} / \mathrm{m}^{2}-\mathrm{h}\right)\end{array}$ \\
\hline ESPA2-LD & 1.25 & 43.65 \\
\hline TMG(D) & 1.14 & 24.7 \\
\hline BW30XFRLE & 1.23 & 39.69 \\
\hline AG LF & 1.15 & 27.02 \\
\hline TM800M & 1.16 & 28.61 \\
\hline SW30XHR & 1.06 & 8.97 \\
\hline
\end{tabular}


Table S2: Experimental and calculated rejections and the mass transfer coefficients for alkanes

\begin{tabular}{|c|c|c|c|c|c|c|c|c|c|c|c|c|c|}
\hline \multirow[t]{2}{*}{ No. } & \multirow[t]{2}{*}{ Compound } & \multicolumn{4}{|c|}{ Toray TMG(D) } & \multicolumn{4}{|c|}{ Dow Filmtec BW30XFRLE } & \multicolumn{4}{|c|}{$\begin{array}{c}\text { Hydranautics } \\
\text { ESPA2-LD }\end{array}$} \\
\hline & & $\operatorname{Rej}_{\text {exp }}, \%$ & $\operatorname{Rej}_{\mathrm{cal}} \%$ & $k_{\text {exp }}$ & $\mathrm{k}_{\mathrm{cal}}$ & $\operatorname{Rej}_{\text {exp }}, \%$ & $\operatorname{Rej}_{c a l} \%$ & $k_{\text {exp }}$ & $\mathrm{k}_{\mathrm{cal}}$ & $\operatorname{Rej}_{\text {expo }} \%$ & $\operatorname{Rej}_{\mathrm{cal}} \%$ & $k_{\text {exp }}$ & $\mathrm{k}_{\mathrm{cal}}$ \\
\hline 1 & Chloroform (CF) & 76.89 & 76.86 & 10.45 & 10.47 & 70.09 & 69.64 & 14.84 & 15.16 & 70.65 & 70.86 & 14.45 & 14.30 \\
\hline 2 & Tetrachloromethane (TCM) & 94.08 & 94.08 & 2.19 & 2.19 & 95.23 & 95.23 & 1.74 & 1.74 & 90.33 & 90.44 & 3.72 & 3.68 \\
\hline 3 & 1,1-Dichloroethane (DCE1) & 80.88 & 82.28 & 8.22 & 7.49 & 77.76 & 85.12 & 9.95 & 6.08 & 78.63 & 86.86 & 9.45 & 5.26 \\
\hline 4 & 1,2-Dichloroethane (DCE3) & 45.57 & 39.65 & 41.55 & 52.94 & 36.52 & 36.10 & 60.45 & 61.57 & 33.05 & 29.52 & 70.47 & 83.03 \\
\hline 5 & 1,1,2-Trichloroethane (TCE4) & 87.12 & 88.29 & 5.14 & 4.61 & 82.52 & 84.86 & 7.37 & 6.20 & 84.56 & 86.63 & 6.35 & 5.37 \\
\hline 6 & 1,1,2,2-Tetrachloroethane (TCE3) & 95.57 & 95.19 & 1.61 & 1.76 & 96.32 & 95.74 & 1.33 & 1.55 & 97.01 & 96.54 & 1.07 & 1.25 \\
\hline 7 & 1,2-Dichloropropane (DCP3) & 90.59 & 90.62 & 3.61 & 3.60 & 88.91 & 92.27 & 4.34 & 2.91 & 90.18 & 93.57 & 3.79 & 2.39 \\
\hline 8 & 1,3-Dichloropropane (DCP4) & 76.45 & 79.19 & 10.71 & 9.14 & 70.72 & 69.74 & 14.40 & 15.09 & 69.02 & 73.96 & 15.61 & 12.24 \\
\hline 9 & 1,2,3-Trichloropropane (TCP1) & 94.03 & 94.01 & 2.21 & 2.22 & 93.27 & 92.12 & 2.51 & 2.97 & 94.49 & 93.45 & 2.03 & 2.44 \\
\hline 10 & Bromoform (BF) & 86.52 & 86.51 & 5.42 & 5.42 & 82.13 & 82.11 & 7.57 & 7.58 & 83.41 & 83.32 & 6.92 & 6.96 \\
\hline 11 & 1,2-Dibromoethane (DBE) & 54.02 & 54.03 & 29.61 & 29.59 & 43.47 & 43.70 & 45.23 & 44.81 & 38.61 & 38.70 & 55.30 & 55.10 \\
\hline 12 & Bromodichloromethane (BDCM) & 83.33 & 83.00 & 6.96 & 7.13 & 80.21 & 80.24 & 8.58 & 8.57 & 80.58 & 80.50 & 8.38 & 8.43 \\
\hline 13 & Chlorodibromomethane & 81.36 & 81.24 & 7.97 & 8.03 & 74.28 & 74.33 & 12.04 & 12.01 & 75.60 & 75.60 & 11.22 & 11.23 \\
\hline 14 & 1,2-Dibromo-3-chloropropane (DCP2) & 95.06 & 95.04 & 1.81 & 1.81 & 95.61 & 95.83 & 1.60 & 1.51 & 96.40 & 96.40 & 1.30 & 1.30 \\
\hline 15 & Isopropyl alcohol (IPA) & 88.13 & 87.38 & 4.68 & 5.02 & 88.73 & 87.77 & 4.42 & 4.85 & 89.69 & 88.29 & 4.00 & 4.61 \\
\hline 16 & Propanone (Acetone) (AT) & 58.34 & 67.59 & 24.84 & 16.68 & 57.16 & 59.51 & 26.06 & 23.67 & 52.96 & 61.03 & 30.90 & 22.21 \\
\hline 17 & 2-Butanone (MEK) & 75.05 & 73.43 & 11.56 & 12.58 & 74.23 & 70.78 & 12.08 & 14.36 & 70.74 & 74.72 & 14.39 & 11.77 \\
\hline 18 & 2-Hexanone (HN) & 84.58 & 82.92 & 6.34 & 7.17 & 85.27 & 86.82 & 6.01 & 5.28 & 80.67 & 91.32 & 8.34 & 3.31 \\
\hline 19 & ethanol & & & -- & -- & 32.19 & 67.14 & 73.26 & 17.03 & 41.78 & 65.67 & 48.47 & 18.19 \\
\hline 20 & ethylene glycol & 44.95 & 61.03 & 42.60 & 22.21 & 55.01 & 74.15 & 28.44 & 12.13 & 63.87 & 70.02 & 19.68 & 14.89 \\
\hline 21 & 1-propanol & & & -- & -- & -- & -- & -- & -- & 70.87 & 81.96 & 14.29 & 7.66 \\
\hline 22 & glycerol & 91.85 & 90.87 & 3.09 & 3.49 & 93.88 & 93.40 & 2.27 & 2.46 & 92.07 & 91.83 & 2.99 & 3.09 \\
\hline 23 & 1-butanol & 69.41 & 69.63 & 15.33 & 15.17 & 81.13 & 84.74 & 8.09 & 6.26 & 81.17 & 87.20 & 8.07 & 5.11 \\
\hline 24 & 2-butanol & 87.41 & 90.17 & 5.01 & 3.79 & 89.24 & 92.21 & 4.19 & 2.94 & 90.78 & 93.43 & 3.53 & 2.44 \\
\hline 25 & isobutanol & & & -- & -- & 94.59 & 91.94 & 1.99 & 3.05 & 95.93 & 92.98 & 1.47 & 2.63 \\
\hline 26 & t-butanol & 88.14 & 87.93 & 4.68 & 4.78 & 90.90 & 93.43 & 3.48 & 2.45 & 92.12 & 93.17 & 2.97 & 2.55 \\
\hline \multirow{2}{*}{ No. } & Comand & \multicolumn{4}{|c|}{ GE AG LF } & \multicolumn{4}{|c|}{ Toray TM800M } & \multicolumn{4}{|c|}{ Dow Filmtec SW30XHR } \\
\hline & Compound & $\operatorname{Rej}_{\text {exp }}, \%$ & $\operatorname{Rej}_{\text {cal }} \%$ & $k_{\text {exp }}$ & $\mathrm{k}_{\mathrm{cal}}$ & $\operatorname{Rej}_{\text {exp }}, \%$ & $\operatorname{Rej}_{\text {cal }} \%$ & $k_{\text {exp }}$ & $\mathrm{k}_{\mathrm{cal}}$ & $\operatorname{Rej}_{\text {exp }}, \%$ & $\operatorname{Rej}_{\mathrm{cal}}, \%$ & $k_{\text {exp }}$ & $\mathrm{k}_{\text {cal }}$ \\
\hline 1 & Chloroform (CF) & 56.52 & 56.59 & 26.76 & 26.68 & 91.64 & 91.64 & 3.17 & 3.17 & 95.61 & 95.61 & 1.60 & 1.60 \\
\hline 2 & Tetrachloromethane (TCM) & 92.48 & 92.49 & 2.83 & 2.83 & 99.06 & 99.05 & 0.33 & 0.33 & 99.35 & 99.35 & 0.23 & 0.23 \\
\hline 3 & 1,1-Dichloroethane (DCE1) & 68.29 & 79.26 & 16.15 & 9.10 & 94.41 & 94.98 & 2.06 & 1.84 & 97.09 & 97.70 & 1.04 & 0.82 \\
\hline 4 & 1,2-Dichloroethane (DCE3) & 28.93 & 22.83 & 85.46 & 117.60 & 76.96 & 74.77 & 10.41 & 11.74 & 88.82 & 88.99 & 4.38 & 4.30 \\
\hline 5 & 1,1,2-Trichloroethane (TCE4) & 73.58 & 75.09 & 12.49 & 11.54 & 95.98 & 95.88 & 1.46 & 1.49 & 97.63 & 97.55 & 0.84 & 0.87 \\
\hline 6 & 1,1,2,2-Tetrachloroethane (TCE3) & 92.73 & 91.89 & 2.73 & 3.07 & 98.51 & 98.46 & 0.53 & 0.54 & 99.19 & 99.14 & 0.28 & 0.30 \\
\hline 7 & 1,2-Dichloropropane (DCP3) & 84.21 & 88.80 & 6.52 & 4.39 & 97.10 & 97.39 & 1.04 & 0.93 & 98.41 & 98.83 & 0.56 & 0.41 \\
\hline 8 & 1,3-Dichloropropane (DCP4) & 52.48 & 59.39 & 31.50 & 23.78 & 90.52 & 93.30 & 3.64 & 2.50 & 94.95 & 95.57 & 1.85 & 1.61 \\
\hline 9 & 1,2,3-Trichloropropane (TCP1) & 88.14 & 86.22 & 4.68 & 5.56 & 98.07 & 97.87 & 0.69 & 0.76 & 98.93 & 98.76 & 0.37 & 0.44 \\
\hline 10 & Bromoform (BF) & 63.01 & 62.92 & 20.42 & 20.50 & 94.68 & 94.73 & 1.96 & 1.94 & 96.70 & 96.69 & 1.19 & 1.19 \\
\hline 11 & 1,2-Dibromoethane (DBE) & 26.42 & 26.93 & 96.89 & 94.37 & 76.80 & 76.78 & 10.51 & 10.52 & 88.31 & 88.32 & 4.60 & 4.60 \\
\hline 12 & Bromodichloromethane (BDCM) & 61.13 & 61.12 & 22.12 & 22.12 & 92.23 & 92.33 & 2.93 & 2.89 & 95.71 & 95.48 & 1.56 & 1.65 \\
\hline 13 & Chlorodibromomethane & 58.40 & 58.16 & 24.77 & 25.03 & 92.86 & 93.03 & 2.67 & 2.60 & 95.66 & 95.65 & 1.58 & 1.58 \\
\hline 14 & 1,2-Dibromo-3-chloropropane (DCP2) & 90.71 & 90.62 & 3.56 & 3.60 & 98.51 & 98.35 & 0.53 & 0.58 & 99.13 & 99.13 & 0.31 & 0.31 \\
\hline 15 & Isopropyl alcohol (IPA) & 81.01 & 80.66 & 8.15 & 8.34 & 96.67 & 96.71 & 1.20 & 1.19 & 98.66 & 98.59 & 0.47 & 0.50 \\
\hline 16 & Propanone (Acetone) (AT) & 39.78 & 56.32 & 52.66 & 26.98 & 84.30 & 84.79 & 6.48 & 6.24 & 93.68 & 93.99 & 2.35 & 2.22 \\
\hline 17 & 2-Butanone (MEK) & 61.93 & 70.14 & 21.39 & 14.81 & 90.90 & 90.19 & 3.48 & 3.78 & 96.41 & 96.05 & 1.29 & 1.43 \\
\hline 18 & 2-Hexanone (HN) & 68.86 & 88.63 & 15.73 & 4.46 & 96.26 & 96.16 & 1.35 & 1.39 & 98.32 & 98.33 & 0.59 & 0.59 \\
\hline 19 & ethanol & 68.96 & 77.17 & 15.66 & 10.29 & -- & -- & -- & -- & -- & -- & -- & -- \\
\hline 20 & ethylene glycol & 82.48 & 89.86 & 7.39 & 3.93 & -- & -- & -- & -- & -- & -- & -- & -- \\
\hline 21 & 1-propanol & 88.94 & 94.36 & 4.32 & 2.08 & -- & -- & -- & -- & -- & -- & -- & -- \\
\hline 22 & glycerol & 96.56 & 96.63 & 1.24 & 1.21 & -- & -- & -- & -- & -- & -- & -- & -- \\
\hline 23 & 1-butanol & 94.29 & 91.82 & 2.11 & 3.10 & -- & -- & -- & -- & -- & -- & -- & -- \\
\hline 24 & 2-butanol & -- & -- & -- & -- & -- & -- & -- & -- & -- & -- & -- & -- \\
\hline 25 & isobutanol & -- & -- & -- & -- & -- & -- & -- & -- & -- & -- & -- & -- \\
\hline 26 & t-butanol & 96.77 & 95.65 & 1.16 & 1.58 & -- & -- & -- & -- & -- & -- & -- & -- \\
\hline
\end{tabular}


Table S3: Experimental and calculated rejections and the mass transfer coefficients for alkenes

\begin{tabular}{|c|c|c|c|c|c|c|c|c|c|c|c|c|c|}
\hline \multirow[t]{2}{*}{ No. } & \multirow[t]{2}{*}{ Compound } & \multicolumn{4}{|c|}{ Toray TMG(D) } & \multicolumn{4}{|c|}{ Dow Filmtec BW30XFRLE } & \multicolumn{4}{|c|}{$\begin{array}{l}\text { Hydranautics } \\
\text { ESPA2-LD }\end{array}$} \\
\hline & & $\operatorname{Rej}_{\exp }, \%$ & $\operatorname{Rej}_{\mathrm{cal}}, \%$ & $k_{\exp }$ & $\mathrm{k}_{\text {cal }}$ & $\operatorname{Rej}_{\exp }, \%$ & $\operatorname{Rej}_{\text {cal, }} \%$ & $k_{\text {exp }}$ & $\mathrm{k}_{\text {cal }}$ & $\operatorname{Rej}_{\exp }, \%$ & $\operatorname{Rej}_{\text {cal, }} \%$ & $\mathrm{k}_{\text {exp }}$ & $\mathrm{k}_{\mathrm{cal}}$ \\
\hline 27 & Trichloroethene (TCE5) & 57.57 & 62.90 & 25.63 & 20.52 & 49.07 & 53.36 & 5.31 & 4.95 & 43.90 & 55.83 & 44.45 & 27.52 \\
\hline 28 & Tetrachloroethene (PCE) & 80.45 & 77.57 & 8.45 & 10.06 & 76.55 & 74.03 & 1.91 & 2.22 & 79.35 & 73.05 & 9.05 & 12.84 \\
\hline 29 & 1,1-Dichloropropene (DCP1) & 57.23 & 57.34 & 25.99 & 25.88 & 46.76 & 46.78 & 4.52 & 4.52 & 43.49 & 42.38 & 45.20 & 47.29 \\
\hline 30 & cis-1,3-Dichloropropene (DCP) & 56.76 & 56.84 & 26.49 & 26.42 & 50.36 & 50.10 & 5.97 & 5.93 & 45.36 & 46.01 & 41.89 & 40.82 \\
\hline 31 & trans-1,3-Dichloropropene (DCP6) & 42.08 & 43.12 & 47.87 & 45.88 & 32.91 & 40.33 & 9.27 & 9.11 & 25.89 & 32.92 & 99.55 & 70.88 \\
\hline 32 & trans-1,4-Dichloro-2-butene (DCB) & 60.68 & 60.14 & 22.54 & 23.06 & 55.07 & 52.36 & 7.06 & 7.14 & 47.65 & 45.29 & 38.21 & 42.01 \\
\hline 33 & Hexachlorobutadiene (HCB) & 96.69 & 96.91 & 1.19 & 1.11 & 95.10 & 95.14 & 0.33 & 0.30 & 94.61 & 96.19 & 1.98 & 1.38 \\
\hline 34 & VA & 60.59 & 59.59 & 22.62 & 23.59 & 49.22 & 48.96 & 3.47 & 3.46 & 37.74 & 35.58 & 57.38 & 62.98 \\
\hline \multirow[t]{2}{*}{ No. } & Compound & \multicolumn{4}{|c|}{ GE AG LF } & \multicolumn{4}{|c|}{ Toray TM800M } & \multicolumn{4}{|c|}{ Dow Filmtec SW30XHR } \\
\hline & & $\operatorname{Rej}_{\text {exp }}, \%$ & $\operatorname{Rej}_{\text {cal }}, \%$ & $k_{\text {exp }}$ & $\mathrm{k}_{\text {cal }}$ & $\operatorname{Rej}_{\exp }, \%$ & $\operatorname{Rej}_{\text {cal }}, \%$ & $k_{\exp }$ & $\mathrm{k}_{\text {cal }}$ & $\operatorname{Rej}_{\text {exp }}, \%$ & $\operatorname{Rej}_{\text {cal }}, \%$ & $k_{\text {exp }}$ & $\mathrm{k}_{\mathrm{cal}}$ \\
\hline 27 & Trichloroethene (TCE5) & 30.86 & 35.93 & 77.91 & 62.03 & 80.21 & 82.44 & 8.58 & 7.41 & 86.75 & 87.54 & 36.10 & 30.40 \\
\hline 28 & Tetrachloroethene (PCE) & 55.19 & 51.23 & 28.24 & 33.11 & 93.48 & 91.43 & 2.43 & 3.26 & 94.81 & 94.01 & 10.66 & 12.20 \\
\hline 29 & 1,1-Dichloropropene (DCP1) & 40.50 & 40.30 & 51.10 & 51.54 & 84.25 & 84.25 & 6.50 & 6.50 & 88.50 & 88.50 & 39.61 & 39.57 \\
\hline 30 & cis-1,3-Dichloropropene (DCP) & 26.98 & 27.12 & 94.14 & 93.46 & 73.44 & 73.47 & 12.58 & 12.56 & 85.35 & 85.44 & 34.29 & 34.64 \\
\hline 31 & trans-1,3-Dichloropropene (DCP6) & -- & -- & -- & -- & 60.80 & 69.87 & 22.42 & 15.00 & 78.95 & 79.24 & 70.92 & 51.47 \\
\hline 32 & trans-1,4-Dichloro-2-butene (DCB) & -- & -- & -- & -- & 74.13 & 72.25 & 12.14 & 13.36 & 83.13 & 82.96 & 28.38 & 31.65 \\
\hline 33 & Hexachlorobutadiene (HCB) & 96.43 & 96.47 & 1.29 & 1.27 & 97.44 & 98.22 & 0.91 & 0.63 & 99.06 & 99.15 & 1.79 & 1.78 \\
\hline 34 & VA & -- & - & - & -- & 79.39 & 79.30 & 9.03 & 9.08 & 90.94 & 90.96 & 35.89 & 36.26 \\
\hline
\end{tabular}


Table S4: Experimental and calculated rejections and the mass transfer coefficients for aromatics

\begin{tabular}{|c|c|c|c|c|c|c|c|c|c|c|c|c|c|}
\hline \multirow[t]{2}{*}{ No. } & \multirow[t]{2}{*}{ Compound } & \multicolumn{4}{|c|}{ Toray TMG(D) } & \multicolumn{4}{|c|}{ Dow Filmtec BW30XFRLE } & \multicolumn{4}{|c|}{$\begin{array}{l}\text { Hydranautics } \\
\text { ESPA2-LD }\end{array}$} \\
\hline & & $\operatorname{Rej}_{\exp }, \%$ & $\operatorname{Rej}_{\text {cal }}, \%$ & $\mathrm{k}_{\exp }$ & $\mathrm{k}_{\mathrm{cal}}$ & $\operatorname{Rej}_{\text {exp }}, \%$ & $\operatorname{Rej}_{\mathrm{cal}}, \%$ & $k_{\text {exp }}$ & $\mathrm{k}_{\text {cal }}$ & $\operatorname{Rej}_{\exp }, \%$ & $\operatorname{Rej}_{\mathrm{cal}}, \%$ & $\mathrm{k}_{\exp }$ & $\mathrm{k}_{\mathrm{cal}}$ \\
\hline 35 & Benzene (BZ) & 82.91 & 82.54 & 7.17 & 7.36 & 79.04 & 79.18 & 9.22 & 9.15 & 76.88 & 77.18 & 10.46 & 10.29 \\
\hline 36 & Toluene (TL) & 85.29 & 81.35 & 6.00 & 7.98 & 81.37 & 76.24 & 7.96 & 10.84 & 79.55 & 73.71 & 8.94 & 12.41 \\
\hline 37 & o-Xylene (OX) & 94.97 & 93.97 & 1.84 & 2.23 & 94.59 & 92.99 & 1.99 & 2.62 & 94.86 & 93.71 & 1.89 & 2.33 \\
\hline 38 & Ethylbenzene (EB) & 88.86 & 88.00 & 4.36 & 4.75 & 84.84 & 84.11 & 6.22 & 6.57 & 85.18 & 83.63 & 6.05 & 6.81 \\
\hline 39 & Vinylbenzene (styrene) (VB) & 81.19 & 81.18 & 8.06 & 8.06 & 73.81 & 73.77 & 12.34 & 12.37 & 71.12 & 71.26 & 14.12 & 14.03 \\
\hline 40 & propylbenzene (PBZ) & 89.31 & 92.49 & 4.16 & 2.82 & 84.67 & 89.72 & 6.30 & 3.99 & 85.71 & 90.30 & 5.80 & 3.74 \\
\hline 41 & Isopropylbenzene (IPB) (Cumene) & 94.25 & 94.27 & 2.12 & 2.11 & 95.99 & 95.95 & 1.45 & 1.47 & 96.77 & 96.38 & 1.16 & 1.30 \\
\hline 42 & 1,2,4-Trimethylbenzene (TEB) & 95.96 & 95.73 & 1.46 & 1.55 & 95.35 & 94.92 & 1.70 & 1.86 & 96.03 & 95.53 & 1.44 & 1.63 \\
\hline 43 & n-Butylbenzene (NBB) & 94.79 & 95.39 & 1.91 & 1.68 & 93.20 & 93.50 & 2.54 & 2.42 & 88.32 & 94.43 & 4.60 & 2.05 \\
\hline 44 & tert-Butylbenzene (TBBZ) & -- & -- & -- & -- & 96.03 & 95.95 & 1.44 & 1.47 & 95.59 & 96.38 & 1.60 & 1.30 \\
\hline 45 & Naphthalene (NP) & 91.25 & 91.33 & 3.34 & 3.30 & 89.91 & 89.69 & 3.90 & 4.00 & 88.95 & 88.94 & 4.32 & 4.32 \\
\hline 46 & Chlorobenzene (CB) & 72.97 & 72.38 & 12.88 & 13.27 & 64.07 & 66.06 & 19.51 & 17.87 & 59.92 & 60.60 & 23.26 & 22.61 \\
\hline 47 & 2-Chlorotoluene (CTL1) & 88.05 & 90.36 & 4.72 & 3.71 & 84.27 & 88.95 & 6.49 & 4.32 & 85.88 & 89.10 & 5.72 & 4.25 \\
\hline 48 & 4-Chlorotoluene (CTL2) & 73.12 & 74.82 & 12.79 & 11.71 & 61.53 & 63.79 & 21.75 & 19.75 & 62.49 & 65.54 & 20.88 & 18.29 \\
\hline 49 & 1,2-Dichlorobenzene (DCB1) & 83.59 & 84.93 & 6.83 & 6.17 & 77.92 & 83.00 & 9.86 & 7.12 & 79.81 & 81.78 & 8.80 & 7.75 \\
\hline 50 & 1,3-Dichlorobenzene (DCB2) & 75.24 & 75.28 & 11.45 & 11.42 & 65.06 & 65.14 & 18.68 & 18.61 & 66.93 & 67.32 & 17.19 & 16.88 \\
\hline 51 & 1,4-Dichlorobenzene (DCB3) & 65.67 & 64.10 & 18.19 & 19.48 & 53.48 & 51.66 & 30.26 & 32.55 & 53.32 & 51.07 & 30.45 & 33.33 \\
\hline 52 & 1,2,3-Trichlorobenzene (TCB1) & 91.24 & 91.12 & 3.34 & 3.39 & 89.36 & 89.22 & 4.14 & 4.20 & 89.28 & 88.30 & 4.18 & 4.61 \\
\hline 53 & 1,2,4-Trichlorobenzene (TCB2) & 83.39 & 82.97 & 6.93 & 7.14 & 78.19 & 80.65 & 9.70 & 8.34 & 74.94 & 77.94 & 11.63 & 9.84 \\
\hline 54 & Bromobenzene (BRB) & 70.24 & 72.18 & 14.74 & 13.41 & 56.12 & 66.06 & 27.19 & 17.87 & 54.51 & 60.60 & 29.02 & 22.61 \\
\hline \multirow[t]{2}{*}{ No. } & Compound & \multicolumn{4}{|c|}{ GE AG LF } & \multicolumn{4}{|c|}{ Toray TM800M } & \multicolumn{4}{|c|}{ Dow Filmtec SW30XHR } \\
\hline & & $\operatorname{Rej}_{\exp }, \%$ & $\operatorname{Rej}_{\text {cal }} \%$ & $\mathrm{k}_{\exp }$ & $\mathrm{k}_{\mathrm{cal}}$ & $\operatorname{Rej}_{\text {exp }}, \%$ & $\operatorname{Rej}_{\text {cal }} \%$ & $k_{\exp }$ & $\mathrm{k}_{\text {cal }}$ & $\operatorname{Rej}_{\exp }, \%$ & $\operatorname{Rej}_{\text {cal }}, \%$ & $k_{\exp }$ & $\mathrm{k}_{\mathrm{cal}}$ \\
\hline 35 & Benzene (BZ) & 68.32 & 68.87 & 16.13 & 15.72 & 94.98 & 95.02 & 1.84 & 1.82 & 97.37 & 97.36 & 0.94 & 0.94 \\
\hline 36 & Toluene (TL) & 67.14 & 59.31 & 17.02 & 23.86 & 95.25 & 93.40 & 1.73 & 2.46 & 97.04 & 96.66 & 1.06 & 1.20 \\
\hline 37 & o-Xylene (OX) & 91.28 & 89.06 & 3.32 & 4.27 & 98.72 & 98.37 & 0.45 & 0.58 & 99.21 & 98.99 & 0.28 & 0.35 \\
\hline 38 & Ethylbenzene (EB) & 75.73 & 73.67 & 11.14 & 12.43 & 96.36 & 97.47 & 1.31 & 0.90 & 97.82 & 98.36 & 0.77 & 0.58 \\
\hline 39 & Vinylbenzene (styrene) (VB) & 57.67 & 58.16 & 25.53 & 25.02 & 92.71 & 92.69 & 2.74 & 2.74 & 95.39 & 95.40 & 1.68 & 1.68 \\
\hline 40 & propylbenzene (PBZ) & 76.40 & 84.30 & 10.75 & 6.48 & 97.38 & 99.05 & 0.94 & 0.33 & 99.22 & 99.20 & 0.27 & 0.28 \\
\hline 41 & Isopropylbenzene (IPB) (Cumene) & 90.93 & 90.93 & 3.47 & 3.47 & 98.93 & 99.32 & 0.38 & 0.24 & 99.46 & 99.61 & 0.19 & 0.14 \\
\hline 42 & 1,2,4-Trimethylbenzene (TEB) & 93.16 & 92.34 & 2.56 & 2.89 & 99.04 & 99.14 & 0.34 & 0.30 & 99.54 & 99.70 & 0.16 & 0.10 \\
\hline 43 & n-Butylbenzene (NBB) & 82.00 & 91.16 & 7.64 & 3.37 & 99.52 & 99.65 & 0.17 & 0.12 & 99.64 & 99.62 & 0.13 & 0.13 \\
\hline 44 & tert-Butylbenzene (TBBZ) & -- & -- & -- & -- & 99.43 & 99.32 & 0.20 & 0.24 & 99.67 & 99.61 & 0.12 & 0.14 \\
\hline 45 & Naphthalene (NP) & 75.85 & 75.80 & 11.08 & 11.11 & 96.80 & 96.80 & 1.15 & 1.15 & 98.69 & 98.56 & 0.46 & 0.51 \\
\hline 46 & Chlorobenzene (CB) & 44.15 & 43.17 & 44.01 & 45.78 & 89.10 & 90.29 & 4.26 & 3.74 & 92.75 & 95.84 & 2.72 & 1.51 \\
\hline 47 & 2-Chlorotoluene (CTL1) & 75.37 & 80.93 & 11.37 & 8.19 & 96.86 & 97.54 & 1.13 & 0.88 & 98.44 & 98.73 & 0.55 & 0.45 \\
\hline 48 & 4-Chlorotoluene (CTL2) & 50.00 & 52.19 & 34.79 & 31.86 & 90.93 & 90.24 & 3.47 & 3.76 & 96.28 & 96.07 & 1.35 & 1.42 \\
\hline 49 & 1,2-Dichlorobenzene (DCB1) & 63.62 & 68.87 & 19.89 & 15.73 & 94.96 & 96.31 & 1.85 & 1.33 & 97.89 & 98.41 & 0.75 & 0.56 \\
\hline 50 & 1,3-Dichlorobenzene (DCB2) & 47.30 & 46.56 & 38.75 & 39.92 & 90.53 & 90.11 & 3.64 & 3.82 & 96.85 & 96.85 & 1.13 & 1.13 \\
\hline 51 & 1,4-Dichlorobenzene (DCB3) & 33.49 & 36.26 & 69.09 & 61.13 & 85.92 & 85.87 & 5.70 & 5.73 & 94.77 & 95.10 & 1.92 & 1.79 \\
\hline 52 & 1,2,3-Trichlorobenzene (TCB1) & 75.97 & 76.26 & 11.00 & 10.83 & 98.36 & 98.35 & 0.58 & 0.58 & 99.54 & 99.54 & 0.16 & 0.16 \\
\hline 53 & 1,2,4-Trichlorobenzene (TCB2) & 59.85 & 63.05 & 23.33 & 20.39 & 97.31 & 97.03 & 0.96 & 1.06 & 99.50 & 99.41 & 0.17 & 0.21 \\
\hline 54 & Bromobenzene (BRB) & 40.57 & 43.67 & 50.96 & 44.86 & 85.82 & 85.80 & 5.75 & 5.76 & 94.08 & 93.32 & 2.19 & 2.49 \\
\hline
\end{tabular}




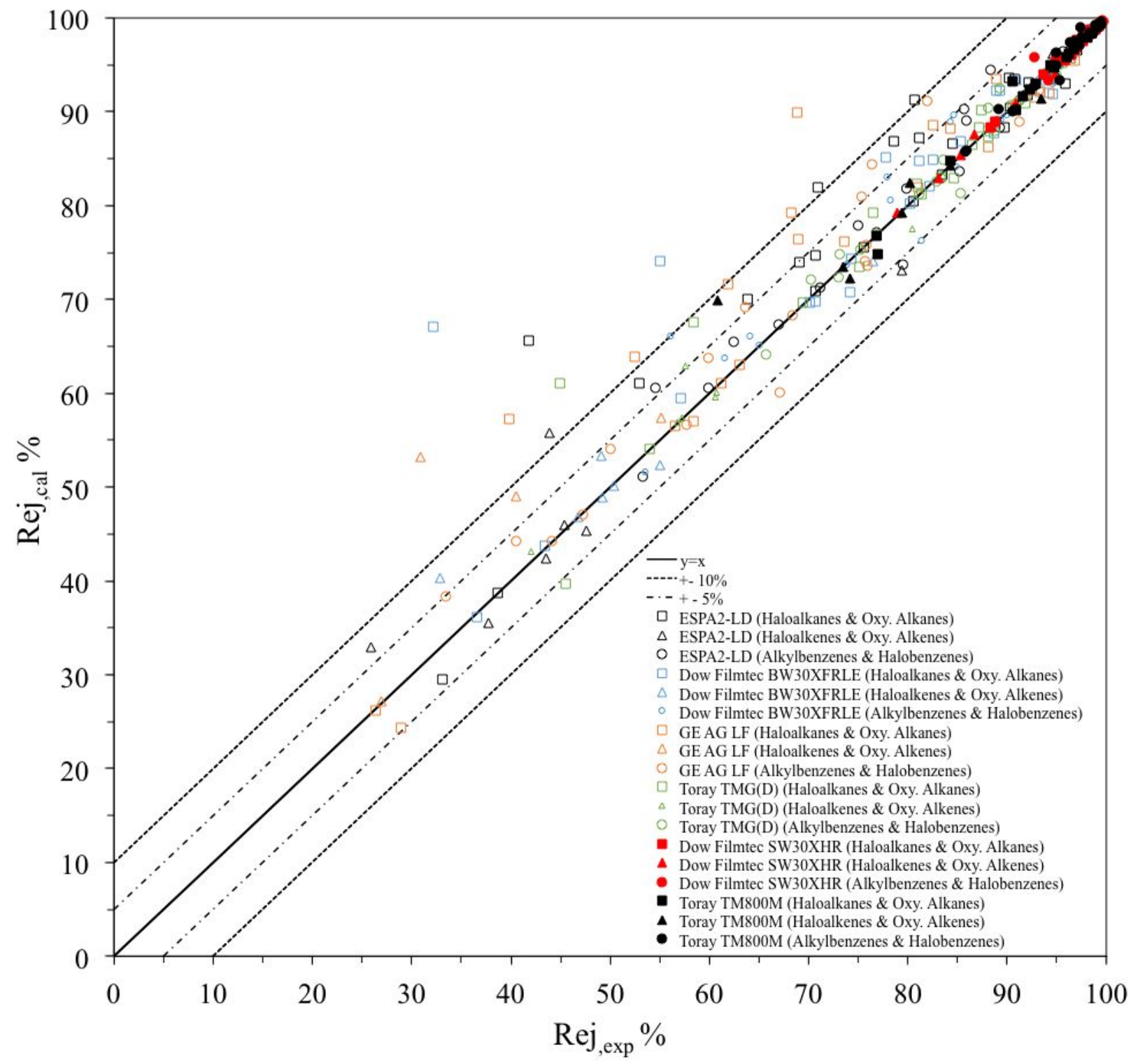

Figure S2: Calculated rejections of organics v.s. experimentally determined rejection of organics through $6 \mathrm{BW}$ and $\mathrm{SW}$ membrane products 


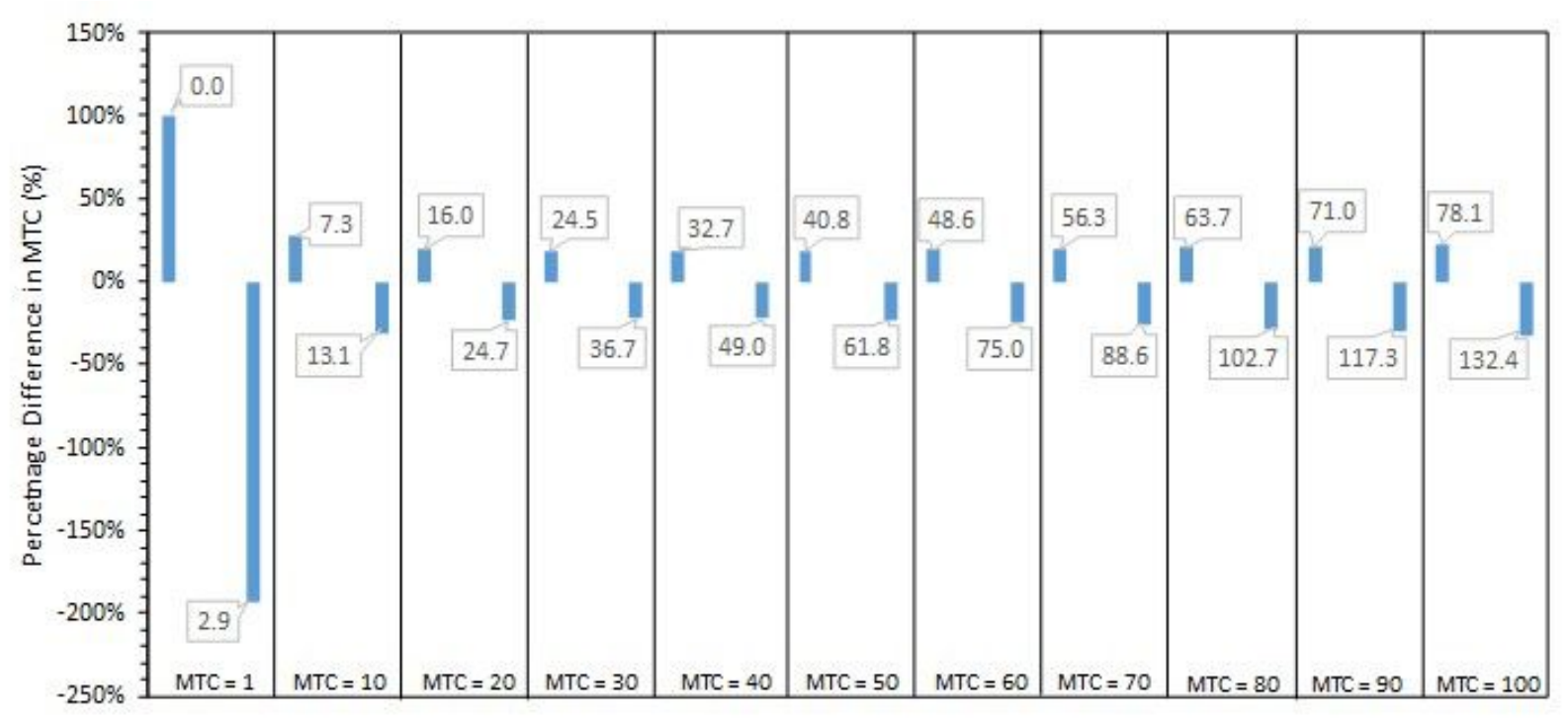

Figure S3: Observed errors in the mass transfer coefficients (MTC) values (i.e., $\mathrm{k}_{\text {Organic }}$ values), caused by $\pm 5 \%$ of experimental errors in the rejection efficiencies for the specific MTC values ranging from 1 to $100 \mathrm{~L} / \mathrm{m}^{2}-\mathrm{h}$.

For example, for the MTC of $10 \mathrm{~L} / \mathrm{m}^{2}-\mathrm{h}, 7.3 \mathrm{~L} / \mathrm{m}^{2}$-h of MTC (i.e., $73 \%$ of $10 \mathrm{~L} / \mathrm{m}^{2}-\mathrm{h}$ ) was the minimum value observed resulting from $+5 \%$ of experimental error in the rejection efficiency and $13.1 \mathrm{~L} / \mathrm{m}^{2}$-h of MTC (i.e., $76 \%$ of $10 \mathrm{~L} / \mathrm{m}^{2}$-h) was the maximum value observed resulting from $-5 \%$ of experimental error in the rejection efficiency. 
Table S5: Experimentally determined rejections of organics found in the literature and the calculated mass transfer coefficients for ESPA2-LD membrane product. All experiments in the literature were conducted at $20-25^{\circ} \mathrm{C}$.

\begin{tabular}{|c|c|c|c|c|c|c|}
\hline Compound & $\begin{array}{l}\text { Molecular } \\
\text { Weight, } \\
\mathrm{g} / \mathrm{mol}\end{array}$ & $\begin{array}{l}\text { Permeate } \\
\text { flux, Jw, } \\
\mathrm{L} / \mathrm{m}^{2}-\mathrm{h}\end{array}$ & $\begin{array}{c}\text { Feed } \\
\text { Concentration, } \\
\mathrm{mg} / \mathrm{L} \\
\end{array}$ & $\mathrm{pH}$ & Rejection, \% & $\begin{array}{l}k_{\text {Organic }}, \\
\mathrm{L} / \mathrm{m}^{2}-\mathrm{h}\end{array}$ \\
\hline $\begin{array}{c}N \text {-ethyl- } N \text { - } \\
\text { methylformamide } \\
\text { (NEMF) }\end{array}$ & 87.1 & 20 & 17 & $6-7$ & $85^{[5]}$ & $3.20^{\mathrm{a}}$ \\
\hline Isobutyraldehyde (IBAL) & 72.1 & 20 & 17 & $6-7$ & $88^{[5]}$ & 2.53 \\
\hline 2-methylbutanal (MBTL) & 86.1 & 20 & 17 & $6-7$ & $91^{[5]}$ & 1.84 \\
\hline $\begin{array}{l}N \text {-nitrosodimethylamine } \\
\text { (NDMA) }\end{array}$ & 74.05 & 20 & $2.5 \times 10^{-4}$ & 8 & $37^{[6]}$ & $30.3^{b}$ \\
\hline $\begin{array}{c}N- \\
\text { nitrosomethylethylamine } \\
\text { (NMEA) }\end{array}$ & 88.06 & 20 & $2.5 \times 10^{-4}$ & 8 & $69^{[6]}$ & $7.98^{\mathrm{b}}$ \\
\hline $\begin{array}{l}N \text {-nitrosopyrrolidine } \\
\text { (NPYR) }\end{array}$ & 100.06 & 20 & $2.5 \times 10^{-4}$ & 8 & $84^{[6]}$ & $3.38^{\mathrm{b}}$ \\
\hline $\mathrm{N}$-nitrosodiethylamine & 102.08 & 20 & $2.5 \times 10^{-4}$ & 8 & $90^{[6]}$ & $1.97^{\mathrm{b}}$ \\
\hline $\begin{array}{l}N \text {-nitrosomorpholine } \\
\text { (NMOR) }\end{array}$ & 116.06 & 20 & $2.5 \times 10^{-4}$ & 8 & $93^{[6]}$ & $1.34^{\mathrm{b}}$ \\
\hline Dichloroacetonitrile & 110 & 18 & 0.05 & 6.8 & $46^{[7]}$ & 19.72 \\
\hline Bromochloroacetonitrile & 154 & 18 & 0.05 & 6.8 & $53^{[7]}$ & 14.66 \\
\hline Dibromoacetonitrile & 199 & 18 & 0.05 & 6.8 & $59^{[7]}$ & 11.17 \\
\hline Dichloroacetamide & 128 & 18 & 0.05 & 6.8 & $80^{[7]}$ & 4.10 \\
\hline Bromochloroacetamide & 172 & 18 & 0.05 & 6.8 & $87^{[7]}$ & 2.40 \\
\hline Dibromoacetamide & 217 & 18 & 0.05 & 6.8 & $88^{[7]}$ & 2.12 \\
\hline
\end{tabular}

a. Pilot-scale experiment using $7.43 \mathrm{~m}^{2}$ of membrane surface area and $9.75 \mathrm{~m}^{3} /$ day of permeate flow

b. Pilot-scale experiment using $4 \mathrm{~mm}$ of hydraulic diameter, $0.1 \mathrm{~m}$ of channel length, $0.42 \mathrm{~m} / \mathrm{s}$ of cross flow velocity, calculated $\beta: 1.05$ and $k_{\mathrm{cp}}=169.8 \mathrm{~L} / \mathrm{m}^{2}-\mathrm{h}$

c. Bench-scale experiment using $1.6 \mathrm{~mm}$ channel height, $139 \mathrm{~cm}^{2}$ of effective membrane area, $0.12 \mathrm{~m} / \mathrm{s}$ of cross flow velocity. 


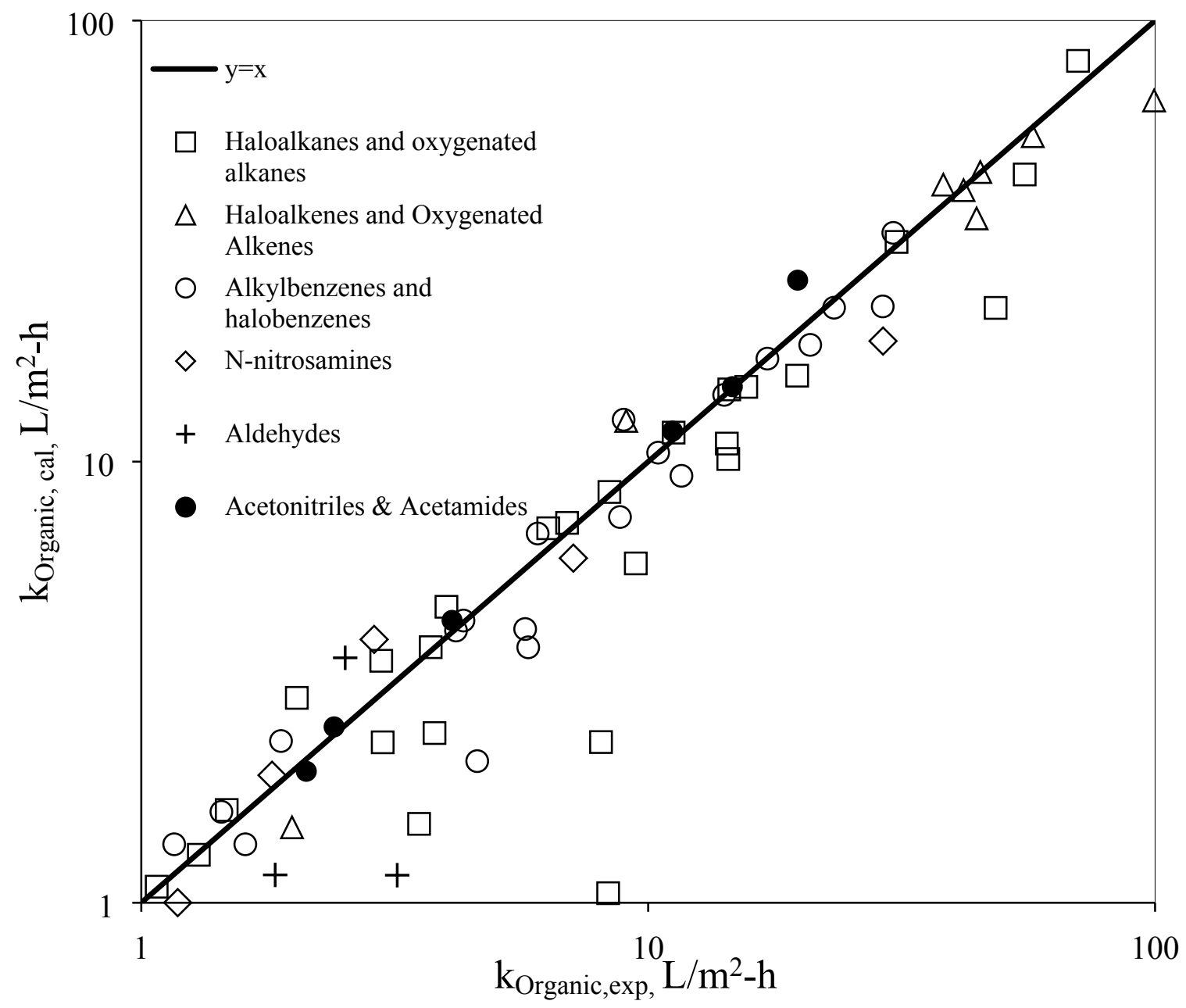

Figure S4: Comparison of experimental and calibrated mass transfer coefficients for expanded GCM for ESPA2-LD membrane. 


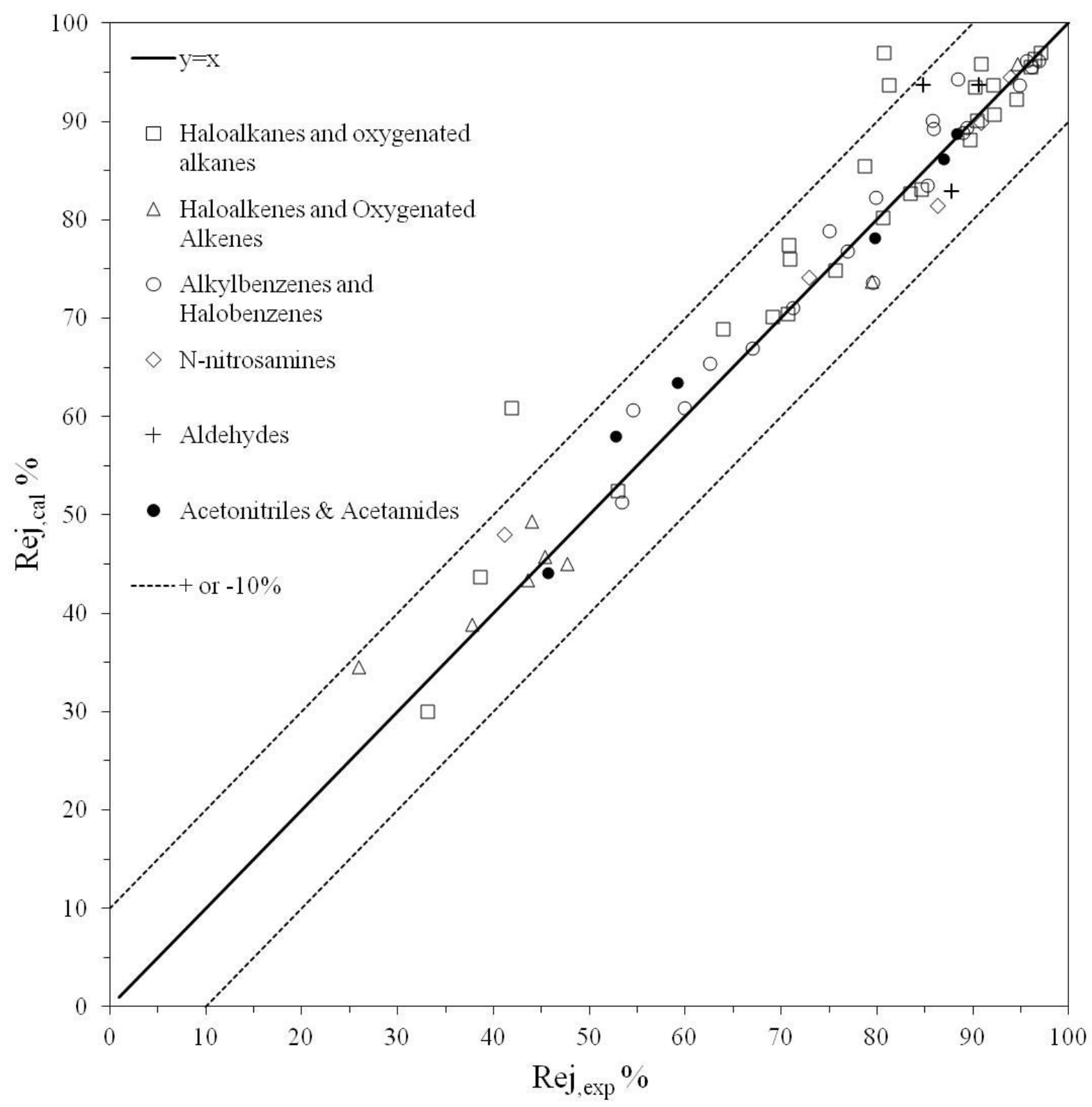

Figure S5: Comparison of experimental and calibrated rejections for expanded GCM for ESPA2LD membrane. 
Text S5: Procedures to calculate the mass transfer coefficients from external experimental data, results and discussions on calibrated parameters and the roles of functional groups on the rejection of organics

The coefficient of $a$ value and the free energies of interaction for each segmented group by the expanded GCM are in Table S5. General trends for alkanes, alkenes, and aromatics were consistent with those in Table 2 . The $k_{\text {Organic }}$ value of acetonitriles (e.g., $25.79 \mathrm{~L} / \mathrm{m}^{2}$-h of dichloroacetonitrile) was determined significantly larger than that of 1,1-dichloroethane (i.e., $\left.5.88 \mathrm{~L} / \mathrm{m}^{2}-\mathrm{h}\right)$ due to the presence of nitrile (-CN) functional group and thus the observed rejection was decreased due to the strong electron negative ability. The $k_{\text {Organic }}$ value of Nnitrosodimethylamine (i.e., $18.77 \mathrm{~L} / \mathrm{m}^{2}$-h) was determined significantly larger than that of isobutyraldehyde (i.e., $3.59 \mathrm{~L} / \mathrm{m}^{2}$-h) due to the presence of nitroso (-NNO) functional group and thus the observed rejection as larger.

For the halogenated acetamides, the presence of $-\mathrm{NH}_{2}$ functional group results in significantly lower the $k_{\text {Organic }}$ value and increased the rejections when compared to the acetonitriles with the same halogenated functional groups. Comparing the $k_{\text {Organic }}$ values of dichloroacetonitrile and dichloroacetamide, the calibrated $k_{\text {Organic }}$ values were 25.79 and 4.34 $\mathrm{L} / \mathrm{m}^{2}$-h, respectively. These results illustrate that the $-\mathrm{NH}_{2}$ functional group has a lower contribution (higher $\Delta G_{\text {solute-membrane }}^{\text {int }}$ ) to the $k_{\text {Organic }}$ value than the - $\mathrm{CN}$ functional group does, resulting in the higher rejections. These results are consistent with the experimental results with the exception of N-ethyl-N-methylformamide, a nitrogenous aldehyde. Comparing this aldehyde with 2-methylbutanal, which only varied by the presence of a $-\mathrm{N}<$ functional group as its base, the experimental results showed that $\mathrm{N}$-ethyl-N-methylformamide should have a higher $k_{\text {Organic }}$ and lower rejection. The GCM determined $k_{\text {Organic }}$ for N-ethyl-N-methylformamide and 2methylbutanal were 1.155 and $1.156 \mathrm{~L} / \mathrm{m}^{2} \mathrm{~h}$, respectively. These results indicate that the presence of nitrogen in place of carbon as a base has little to no impact on the $k_{\text {Organic }}$ value and the 
rejection efficiency, where the experimental results show a difference of only $6 \%$ in the rejection efficiency. 
Table S6: Coefficients and free energies of interaction determined by an upgraded GCM for the ESPA2-LD membrane product.

\begin{tabular}{|c|c|c|}
\hline Group & Parameters & Value \\
\hline \multirow{25}{*}{$\begin{array}{l}\mathrm{A} \\
\mathrm{l} \\
\mathrm{k} \\
\mathrm{a} \\
\mathrm{n} \\
\mathrm{e} \\
\mathrm{s}\end{array}$} & $\bar{a}$ & 623 \\
\hline & $-\mathrm{CH}_{3}$ & 1.488 \\
\hline & $-\mathrm{CH}_{2^{-}}$ & 1.133 \\
\hline & -CHCl- & 2.757 \\
\hline & $-\mathrm{CH}_{2} \mathrm{Cl}$ & 1.304 \\
\hline & $-\mathrm{CH}_{2} \mathrm{Cl}\left(2^{\text {nd }}\right)$ & 0.736 \\
\hline & $-\mathrm{CHCl}_{2}$ & 3.175 \\
\hline & $-\mathrm{CCl}_{3}{ }^{2}$ & 3.755 \\
\hline & $-\mathrm{CCl}_{4}$ & 5.100 \\
\hline & -CHBr- & 3.564 \\
\hline & $-\mathrm{CH}_{2} \mathrm{Br}$ & 1.316 \\
\hline & $-\mathrm{CHClBr}$ & 3.731 \\
\hline & $-\mathrm{CHBr}_{2}$ & 3.963 \\
\hline & $-\mathrm{CBr}_{3}$ & 4.451 \\
\hline & $-\mathrm{CCl}_{2} \mathrm{Br}$ & 4.290 \\
\hline & $-\mathrm{CClBr}_{2}$ & 3.980 \\
\hline & $-\mathrm{CH}(\mathrm{OH})-$ & 1.914 \\
\hline & $>\mathrm{CO}$ & $9.39 \times 10^{-3}$ \\
\hline & $-\mathrm{OH}$ & 0.708 \\
\hline & $-\mathrm{CHO}$ & 2.180 \\
\hline & $-\mathrm{O}-$ & 1.374 \\
\hline & -N-NO & 0.527 \\
\hline & $-\mathrm{N}<$ & $1.54 \times 10^{-3}$ \\
\hline & $-\mathrm{CN}$ & 0.275 \\
\hline & $-\mathrm{NH}_{2}$ & 1.776 \\
\hline \multirow{8}{*}{$\begin{array}{l}\mathrm{A} \\
1 \\
\mathrm{k} \\
\mathrm{e} \\
\mathrm{n} \\
\mathrm{e} \\
\mathrm{s}\end{array}$} & $a^{\prime}$ & 855 \\
\hline & $>\mathrm{C}=\mathrm{C}<$ & $7.65 \times 10^{-3}$ \\
\hline & $>\mathrm{C}=\mathrm{C}<$ (cis) & 0.478 \\
\hline & $>\mathrm{C}=\mathrm{C}<$ (trans) & $9.89 \times 10^{-3}$ \\
\hline & $-\mathrm{CH}_{3}$ & 0.594 \\
\hline & $-\mathrm{Cl}$ & 1.057 \\
\hline & $-\mathrm{CH}_{2} \mathrm{Cl}$ & 1.496 \\
\hline & $-\mathrm{COO}-$ & 1.254 \\
\hline \multirow{14}{*}{$\begin{array}{l}\text { i } \\
c \\
\text { s }\end{array}$} & $a "$ & 34 \\
\hline & $\mathrm{C}_{6} \mathrm{H}_{6}$ & 1.170 \\
\hline & $\mathrm{C}_{6} \mathrm{H}_{5}$ & 0.404 \\
\hline & $\mathrm{C}_{6} \mathrm{H}_{4}(1,2)$ & 1.487 \\
\hline & $\mathrm{C}_{6} \mathrm{H}_{4}(1,3)$ & 0.660 \\
\hline & $\mathrm{C}_{6} \mathrm{H}_{4}(1,4)$ & $3.60 \times 10^{-3}$ \\
\hline & $\mathrm{C}_{6} \mathrm{H}_{3}(1,2,3)$ & 2.070 \\
\hline & $\mathrm{C}_{6} \mathrm{H}_{3}(1,2,4)$ & 1.262 \\
\hline & $-\mathrm{CH}_{3},-\mathrm{CH}_{2-}$ & 0.594 \\
\hline & $>\mathrm{C}<,-\mathrm{CH}<$ & 0.464 \\
\hline & $-\mathrm{C}=\mathrm{Cl}<$ & 2.810 \\
\hline & $-\mathrm{Cl}$ & $9.45 \times 10^{-3}$ \\
\hline & $-\mathrm{Br}$ & $2.66 \times 10^{-3}$ \\
\hline & Naphthalene & 2.046 \\
\hline
\end{tabular}




\section{Text S6: Evaluation of GCM parameters and roles of functional groups in interaction.}

The correlation between $\sigma^{*}$ and $\Delta G_{\text {solute-membrane }}^{\text {intr }}$ values indicates that the increasing electron withdrawing ability (i.e., higher $\sigma^{*}$ ) associated with increasing number of chlorine or bromine atoms likely increases the interaction with the membrane. A strong correlation between steric constants and $\Delta G_{\text {solute-membrane }}^{\text {intr }}$ values was also observed for both chlorine and bromine, indicating that the size of these halogenated functional group contribute to the interaction between the functional group and membrane. The nitrile $(-\mathrm{CN})$ functional group has a strong electron withdrawing ability (i.e., high $\sigma^{*}$ ), however: the interaction with the membrane is low (0.275). The cause of the low interaction with the membrane is likely due to the small molecular size of the - $\mathrm{CN}$ functional group, which contains a short carbon-nitrogen triple bond and is highly polar. The $-\mathrm{CN}$ functional group has a steric constant of -0.51 , indicating weak steric effects which is supportive of low membrane interaction due to small molecular size. While no Taft constant is available for the -N-NO functional group, it is expected to have a high electron withdrawing ability and similar to $-\mathrm{CN}$, the interaction with the membrane is relatively low (0.527). Previous studies have found that the rejection of N-nitrosamines is largely governed by size-based exclusions ${ }^{[5]}$, which is consistent with a low $\Delta G_{\text {solute-membrane }}^{\text {intr }}$ value for the $-\mathrm{N}-\mathrm{NO}$ functional group. The $-\mathrm{NH}_{2}$ functional group has a strong electron donating ability and weak steric effects (i.e., low $\sigma^{*}$ and high $\mathrm{E}_{\mathrm{S}}$ ). However, the interaction with the membrane is relatively high (1.776). The membrane consists of an ultrathin polyamide active skin layer, ${ }^{[7]}$ containing amine $\left(-\mathrm{NH}_{2}\right)$ and keto $(=\mathrm{O})$ functional groups, which may increase the interaction of the $-\mathrm{NH}_{2}$ functional group. Both the $-\mathrm{NH}_{2}$ functional group and the active skin layer have the ability to form hydrogen-bonds (H-bonds) ${ }^{[7]}$ and may play a role in the increased interaction of the amine functional group. Generally, a trend can be observed that nitrogen-containing organics tend to 
have lower rejections than organics containing the same or similar functional groups without nitrogen. Nitrogen atoms in molecules can act as H-bond acceptors, which can be formed with the active skin layer of the membrane and consequently facilitate their adsorption to the membrane or diffusion to the permeate, decreasing the rejection of nitrogen-bearing compounds..$^{[5,7]}$
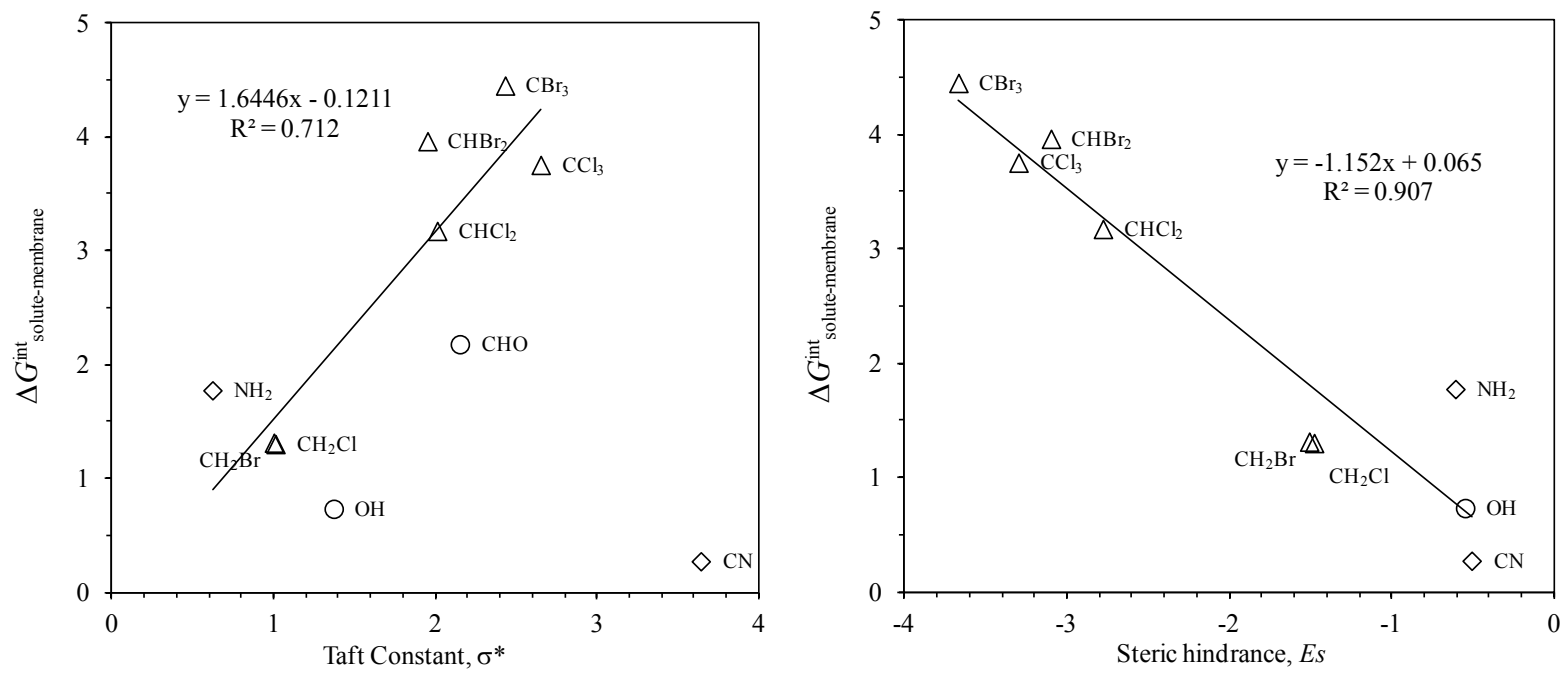

Figure S6: Correlations between the free energies of interaction and Taft constant and Steric hindrance, respectively.

Table S7: Values for comparison of $\Delta G_{\text {solute-membrane }}^{\text {int }}$, Taft constants, and steric hindrance. Taft and steric hindrance constants were obtained from [9].

\begin{tabular}{|c|c|c|c|}
\hline Functional Group & $\Delta G_{\text {solute-membrane }}^{\text {intr }}$ & Taft Constant $\left(\sigma^{*}\right)$ & Steric Hindrance $\left(\mathrm{E}_{\mathrm{S}}\right)$ \\
\hline$-\mathrm{OH}$ & 0.736 & 1.37 & -0.55 \\
$-\mathrm{CHO}$ & 2.180 & 2.15 & - \\
$-\mathrm{NH} H_{2}$ & 2.180 & 0.62 & -0.61 \\
$-\mathrm{CN}$ & 0.275 & 3.64 & -0.51 \\
$-\mathrm{CH}_{2} \mathrm{Cl}$ & 1.304 & 1.01 & -1.48 \\
$-\mathrm{CHCl}_{2}$ & 3.175 & 2.01 & -2.78 \\
$\mathrm{CCl}_{3}$ & 3.755 & 2.65 & -3.30 \\
$-\mathrm{CH}_{2} \mathrm{Br}$ & 1.316 & 1.00 & -1.51 \\
$-\mathrm{CHBr}_{2}$ & 3.963 & 1.95 & -3.10 \\
$\mathrm{CBr}_{3}$ & 4.451 & 2.43 & -3.67 \\
\hline
\end{tabular}


Table S8: Results of prediction of organic rejection through ESPA2-LD under different operational conditions as well as through DOW Filmtec BW30-XFRLE membrane, using ESPA2-LD as a reference membrane.

\begin{tabular}{|c|c|c|c|c|}
\hline Compound & Membrane & $\begin{array}{l}\text { Experimental } \\
\text { Rejection (\%) }\end{array}$ & $\begin{array}{c}\text { Predicted } \\
\text { Rejection }(\%)\end{array}$ & $\%$ Difference \\
\hline NDMA & $\begin{array}{c}\text { Filmtec BW30- } \\
\text { XFRLE }\end{array}$ & $61^{[10]}$ & 61.8 & 1.34 \\
\hline NMEA & $\begin{array}{c}\text { Filmtec BW30- } \\
\text { XFRLE }\end{array}$ & $85^{[10]}$ & 85.8 & 0.93 \\
\hline NPYR & $\begin{array}{c}\text { Filmtec BW30- } \\
\text { XFRLE }\end{array}$ & $94^{[10]}$ & 90.5 & 3.77 \\
\hline NDEA & $\begin{array}{c}\text { Filmtec BW30- } \\
\text { XFRLE }\end{array}$ & $97^{[10]}$ & 95.18 & 1.87 \\
\hline Chloroform & ESPA2-LD & $53^{[11]}$ & 53.2 & 0.372 \\
\hline 1,2-Dichloropropane & ESPA2-LD & $89^{[11]}$ & 88.0 & 1.127 \\
\hline 1,1,2-trichloroethane & ESPA2-LD & $84^{[11]}$ & 71.0 & 15.444 \\
\hline Bromodichloromethane & ESPA2-LD & $67^{[11]}$ & 66.8 & 0.304 \\
\hline
\end{tabular}

\section{References}

1. Breitner, N. L.; Howe, J. K.; Minakata, D. Boron can be used to predict trace organic rejection through reverse osmosis membranes for potable reuse. Environ. Sci. Technol. 2018, 52, 13871-13878.

2. $\quad$ U.S. EPA. 2003. "Method 5030C (SW-846): Purge-and-Trap for Aqueous Samples," Revision 3. Washington, DC.

3. U.S. EPA. 2006. "Method 8260D (SW-846): Volatile Organic Compounds by Gas Chromatography/Mass Spectrometry (GC/MS)," Revision 3. Washington, DC.

4. $\quad$ Crittenden, J.C.; Trussell, R.R.; Hand, D.W.; Howe, K.J.; Tchobanoglous, G. MWH's Water Treatment: Principles and Design, 3rd edition. 2012. MWH.

5. $\quad$ Fujioka, T.; Kodamatani, H.; Nghiem, L. D.; Shintani, T. Transport of N-Nitrosamines through a Reverse Osmosis Membrane: Role of Molecular Size and Nitrogen Atoms. Environmental Science \& Technology Letters 2019, 6 (1), 44-48.

6. Fujioka, T.; Oshima, N.; Suzuki, R.; Khan, S. J.; Roux, A.; Poussade, Y.; Drewes, J.E.; Ngheim, L.D. Rejection of small and uncharged chemicals of emerging concern by reverse osmosis membranes: The role of free volume space within the active skin layer. Separation and Purification Technology. 2013, 116, 426-432.

7. $\quad$ Doederer, K.; Farré, M. J.; Pidou, M.; Weinberg, H. S.; Gernjak, W. Rejection of Disinfection by-Products by RO and NF Membranes: Influence of Solute Properties and Operational Parameters. Journal of Membrane Science 2014, 467, 195-205.

8. Mariňas, B. J.; Urama, R.I. Modeling concentration-polarization in reverse osmosis spiral-wound elements. J. Env. Eng. 1996, 122, 4, 292-298. 
9. $\quad$ Karelson, M. Molecular descriptors in QSAR/QSPR; Wiley-Interscience: New York, 2000.

10. Steinledarling, E.; Zedda, M.; Plumlee, M.; Ridgway, H.; Reinhard, M. Evaluating the Impacts of Membrane Type, Coating, Fouling, Chemical Properties and Water Chemistry on Reverse Osmosis Rejection of Seven Nitrosoalklyamines, Including NDMA. Water Research 2007, 41 (17), 3959-3967.

11. Takeuchi, H.; Tanaka, H.; Nghiem, L. D.; Fujioka, T. Emerging Investigators Series: a Steric Pore-Flow Model to Predict the Transport of Small and Uncharged Solutes through a Reverse Osmosis Membrane. Environmental Science: Water Research \& Technology 2018, 4 (4), 493-504. 